\title{
An Edge-Preserved Image Denoising Algorithm Based on Local Adaptive Regularization
}

\author{
Li Guo, ${ }^{1}$ Weilong Chen, ${ }^{2}$ Yu Liao, ${ }^{1}$ Honghua Liao, ${ }^{1}$ and Jun Li ${ }^{1}$ \\ ${ }^{1}$ Information Engineering Department, Hubei University for Nationalities, Enshi 445000, China \\ ${ }^{2}$ Digital Media College, Sichuan Normal University, Chengdu 610068, China \\ Correspondence should be addressed to Weilong Chen; 312790467@qq.com
}

Received 19 March 2015; Revised 1 July 2015; Accepted 12 July 2015

Academic Editor: Marco Anisetti

Copyright (c) $2016 \mathrm{Li}$ Guo et al. This is an open access article distributed under the Creative Commons Attribution License, which permits unrestricted use, distribution, and reproduction in any medium, provided the original work is properly cited.

\begin{abstract}
Image denoising methods are often based on the minimization of an appropriately defined energy function. Many gradient dependent energy functions, such as Potts model and total variation denoising, regard image as piecewise constant function. In these methods, some important information such as edge sharpness and location is well preserved, but some detailed image feature like texture is often compromised in the process of denoising. For this reason, an image denoising method based on local adaptive regularization is proposed in this paper, which can adaptively adjust denoising degree of noisy image by adding spatial variable fidelity term, so as to better preserve fine scale features of image. Experimental results show that the proposed denoising method can achieve state-of-the-art subjective visual effect, and the signal-noise-ratio (SNR) is also objectively improved by $0.3-0.6 \mathrm{~dB}$.
\end{abstract}

\section{Introduction}

In the process of image acquisition and transmission, all recording devices have traits which make them susceptible to noise. Noise deteriorates the quality of image and causes difficulty in image observation, feature extraction, and image analysis. In order to effectively reduce noise, some filters such as mean filter and Gaussian filter are applied on the noisy image. It is limited that filters can lose large edge and texture information of image in denoising process. In order to avoid this problem, many researchers have been working on different denoising methods. Weickert proposed a partial differential equation (PDE) method which can effectively remove noise [1]. Chan and Esedoglu proposed a total variation method based on L1 norm; it produced stair effect in smoothing area [2]. Bo and Li used a symmetric four-order PDE method to achieve acceptable denoising result [3]. Xu and Wang introduced nonlocal means into regularization to obtain a denoising version; this method oversmoothed image edges and details [4]. Gupta and Kumar proposed a generalized total variation denoising model, which can remove false edge, but it is sensitive to the selection of $P$ factor [5]. Liu and
Zeng proposed a map image adaptive regularization denoising method to get a good visual effect [6]. These methods can improve the image denoising quality in some extent but destroy high-frequency information of image inevitably. In recent years, Liu and Huang proposed a new nonlocal total variation regularization algorithm for image denoising [7]. Chen et al. proposed an adaptive denoising model by regulating regularization and fidelity total variation [8]. Suman used adaptive median filter into image denoising and got good result [9]. Yan and Lu added least squares fidelity in imaging denoising by generalized total variation regularization [10]. Anilet and Hati combined curvelet transform and wiener filter to effectively reduce noise in an image [11]. Liu et al. discussed many methods in image fusion and image denoising, which all are useful and popular [12]. Abovementioned image denoising methods achieve acceptable denoising effect, but the selection of an appropriate regularized factor and reasonable iteration is still a problem. For this reason, the goal of this study is to examine efficient and reliable image denoising algorithms. This paper proposed an image denoising method based on local adaptive regularization, which can adaptively adjust denoising according to different area of noisy image 
and better protect the texture and details of image, so as to achieve state-of-the-art denoising effect.

The rest of this paper is organized as follows. The introduction of regularization (especially for total variation regularization) is described in Section 2. Further, Section 3 details main idea of the proposed method in this paper. And experimental results are presented in Section 4. Finally, summary and outlook are discussed in Section 5, that is, the conclusion of this paper.

\section{Total Variation Denoising}

In signal processing, total variation denoising is remarkably effective at simultaneously preserving edges whilst smoothing away noise in flat regions, even at low signal-to-noise ratios. It is based on the principle that signals with excessive and possibly spurious detail have high total variation (TV); that is, the integral of the absolute gradient of the signal is high. According to this principle, reducing the total variation of the signal subject to its being a close match to the original signal removes unwanted detail whilst preserving important details such as edges. The concept of total variation was pioneered by Rudin et al. [13]. In area of image processing, suppose $f$ is the original image, $f_{0}$ is the noisy version of $f$, and this relation can be mathematically expressed by

$$
f_{0}(x, y)=f(x, y)+n(x, y) .
$$

Here $n(x, y)$ is random noise with zero mean and $\sigma^{2}$ variation. At the same time, we can, for example, define the total variation as $V(x, y)=\sum_{n}\left|f^{n+1}(x, y)-f^{n}(x, y)\right|$; the goal of TV denoising is to find an approximation, which is smaller but close to the one before. That is, TV denoising is a minimization process; it explores the equal state of energy function relative to the TV norm of $f$ and the regularization of $f_{0}$, expressed in

$$
E_{\mathrm{TV}}=\int_{\Omega}\left[(|\nabla f|)+\frac{1}{2} \lambda\left(f-f_{0}\right)^{2}\right] d x d y .
$$

Here $\Omega$ represents the domain of image, all pixels $(x, y) \epsilon$ $\Omega$. Normally the TV of ideal image is smaller than noisy image, so minimizing TV can reduce the noise of image. Based on this principle, (2) can be equaled as follows:

$$
\frac{\lambda}{2} \int_{\Omega}\left(f-f_{0}\right)^{2} d x d y+\int_{\Omega} \sqrt{f_{x}^{2}+f_{y}^{2}} d x d y=0 .
$$

The first term in (3) is data fidelity term, which can retain characteristics of the original image and reduce distortion. The second term in (3) is regularization term, which depends on noise level and balance denoising and smoothing. The Euler-Lagrange equation derivate from (3) is represented by

$$
\lambda\left(f-f_{0}\right)-\nabla \cdot\left(\frac{\nabla f}{|\nabla f|}\right)=0 .
$$

Here $1 /|\nabla f|$ is a diffusion coefficient. In edge of image, large $\nabla f$ will lead to small diffusion coefficient, so the diffusion along edge is weak to preserve edge of image. In smoothing area of image, small $|\nabla f|$ will lead to large diffusion coefficient, so the diffusion in smoothing area is strong to remove noise in image. The regularization parameter $\lambda$ plays a critical role in the denoising process. When $\lambda=0$, there is no denoising and the result is identical to the input signal. As $\lambda \rightarrow \infty$, however, the total variation term plays an increasingly strong role, which forces the result to have smaller total variation, at the expense of being less like the input (noisy) signal. Thus, the choice of regularization parameter is critical to achieving just the right amount of noise removal.

\section{Image Denoising Method Based on Local Adaptive Regularization}

Referring to the classical TV model which described in Section 2, it can reduce noise by energy constraint, however, the selection of an appropriate regularization factor is also a difficult problem. High $\lambda$ brings oversmoothing and small $\lambda$ reduces noise ineffectively. In order to handle impulsive noisy image, an appropriate regularization factor $\lambda$ must be obtained to reduce different noise in image, which aims at balance of data fidelity term and regularization term. For these discussions, a novel image denoising method based on local adaptive regularization is proposed here. According to noise level in different image area, it defines a space variable energy function and can adaptively adjust denoising degree. In order to clearly elaborate idea, the main steps described are as follows.

Step 1 (global residual noisy energy computation). This step produces the residual error of noise in input image. Suppose input noisy image is $f_{0}(x, y)$. Estimated denoising version of $f_{0}(x, y)$ is obtained by classical TV regularization, which is expressed as $f_{0}^{\prime}(x, y)$. Then, global residual noisy energy $f_{r}$ can be computed by expression $f_{r}=f_{0}(x, y)-f_{0}^{\prime}(x, y)$. Therefore, the mean value of global residual error $f_{r}$ is computed and named as $M_{r}$.

Step 2 (local energy computation). On the basis of first step, local variance of residual image can be expressed by $P_{\mathrm{LV}}(x$, $y)=(1 /|\Omega|) \int_{\Omega} f_{r}(x, y) w(x, y) d x d y$, where $w(x, y)$ is a normalized and radial symmetric smoothing-window and $\int_{\Omega} w(x, y) d x d y=1$. Suppose $S(x, y)=\sigma^{4} / P_{\mathrm{LV}}$ to get prior information of noise energy in noisy image; here $\sigma$ is noise standard deviation of input noisy image $f_{0}(x, y)$.

Step 3 (iteratively compute regularization factor to achieve local adaptive regularization). Define $n=1$ and suppose $f^{(n)^{\prime}}=f_{0}^{\prime}(n=1)$. Compute $Q^{n}=\left(f^{(n)^{\prime}}-f_{0}\right) \operatorname{div}\left(\phi^{\prime}\left(\nabla f^{(n)^{\prime}} /\right.\right.$ $\left.\left.\left|f^{(n)^{\prime}}\right|\right)\right)$; regularization factor $\lambda$ is then computed by $\lambda^{n}(x, y)=Q^{n}(x, y) / S(x, y)$. In this way, each iteration of $\lambda$ can be applied to (4) to obtain an estimated denoising version. When condition of convergence is satisfied, the final denoising result can be achieved.

\section{Experiments and Discussion}

In this section, we validate the potential of the proposed method by simulated noisy image experiment and real noisy 


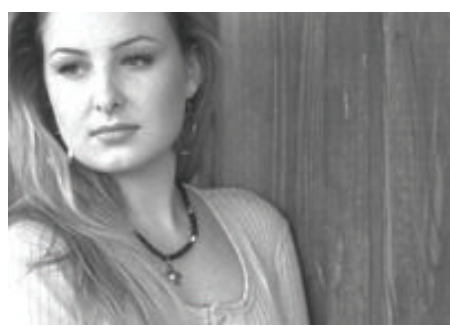

(a) Original image

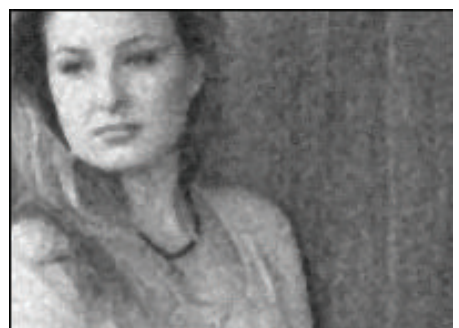

(d) Bilateral filter denoising

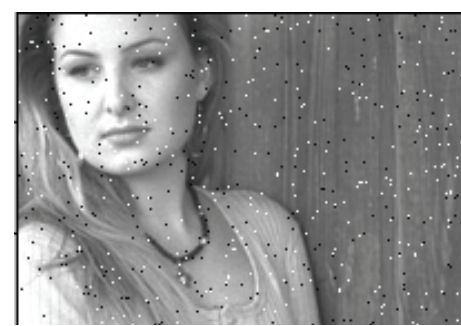

(b) Noisy image

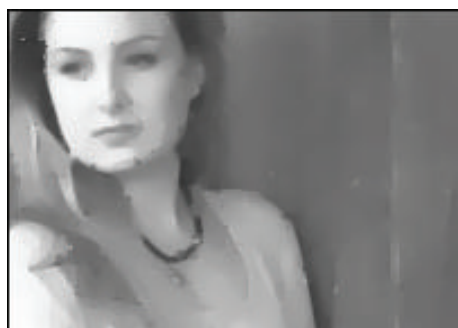

(e) The proposed method in [4]

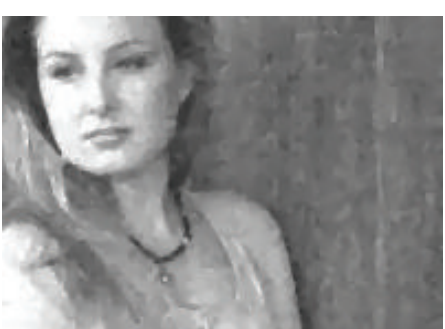

(c) Classical TV denoising

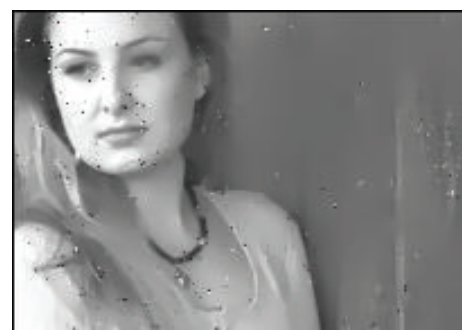

(f) The proposed method in this paper

FIGURE 1: Denoising comparison of salt and pepper noisy image. (The SNR value of this comparison is as follows: noisy image SNR $=8.1867$, classical TV denoising SNR $=15.2426$, bilateral filter denoising SNR $=15.0410$, the proposed method in [4] SNR $=15.7141$, and the proposed method in this paper SNR $=15.9832$.)

image experiment. The comparison we provide here is the denoising results by classical TV denoising, bilateral filtering, and the denoising method proposed in [4].

\subsection{Simulated Noisy Image}

4.1.1. Salt and Pepper Noise. Firstly, the test image woman was additive with salt and pepper noise, at which variance is 0.02. In order to better show different denoising comparison, experimental result shown here is the enlarged local area of woman.

Figure 1(a) is the clear original woman image, Figure 1(b) is seen to contain a large number of salt and pepper noises and many noise points contaminate image, Figure $1(\mathrm{c})$ is the denoising result of classical TV method, here texture in the right smoothing part of image is preserved, and conversely woman's face detailed information is lost and edge of image is not ideal, Figure 1(d) is the denoising result by bilateral filter, which is more fuzzy and noisy, and the visual effect is bad, and Figure 1(e) is the denoising result by method in [4] and noise is removed in this result; however the overall effect is oversmoothing. The denoising result in Figure 1(f) is clear and details are preserved well, although there are still a few grain noises, but it does not affect the general viewing effect.

4.1.2. Gaussian Noise. In this experiment, test image Lena is added into Gaussian noise with 0 mean and variation is 0.06 . As the same way to experiment 1, Figure 2 also shows the extracted local Lena's face. From this way, the different denoising result is compared more clearly here.

When compared to these different denoising methods, Figure 2(a) is the local part of the original Lena image, Figure 2(b) is local part of noisy image added with Gaussian noise and a large number of noises can be seen in it, Figure 2(c) is the local part of denoising result by classical TV method and the visual effect is bad, Figure $2(\mathrm{~d})$ is the denoising result of bilateral filter and it also contains quite a few noises in denoising result, and Figure 2(e) is the denoising result of method in [4] and it well reduces noise in noisy image, but the detailed information is lost and overall appearance is too smoothing. Figure 2(f) is the local part of denoising result by the proposed method in this paper, it can be seen that the detailed information (such as feather in cap, eye, and mouth edge) is kept well, the people's viewing effect is very good, and noise is reduced selectively in a natural manner.

4.1.3. Random Noise. In order to further comparison of denoising method, standard test image Lena is added into random noise with variance being 20 . From this way, a simulated noisy image is obtained in this experiment. For better illustrating the effectiveness of the proposed algorithm, the local part of denoising result is extracted to be shown in Figure 3.

Figures 3(a) and 3(b) show the local part of original version and noisy version of Lena image, and the noisy version is addictive with random noise. Figure 3(c) is the local part of denoising result by classical TV method and it looks more fuzzy, Figure 3(d) maintains a noisy state, which contains a large number of unremoved noises, Figure 3(e) is the local part of denoising result by denoising method in [4] and it can effectively reduce noise but smoothed the details simultaneously, and Figure 3(f) is the local part of denoising result by the proposed method in this paper, it can be seen that the detailed information is protected well (such as eye and the edge of mouth), and this visual effect is much acceptable and outweighs other denoising results. 


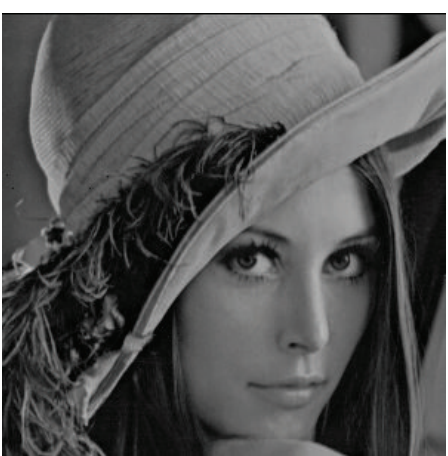

(a) Original Lena image

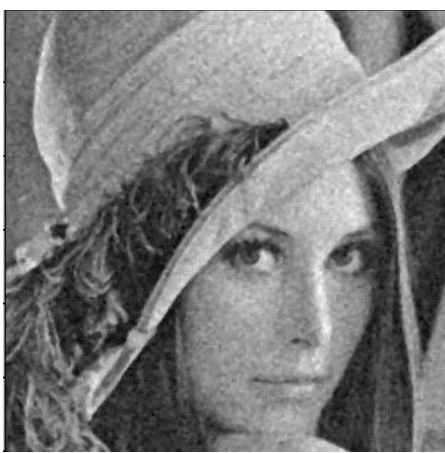

(d) Bilateral filter denoising

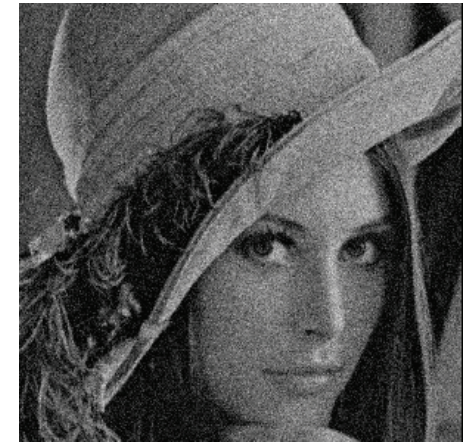

(b) Noisy image

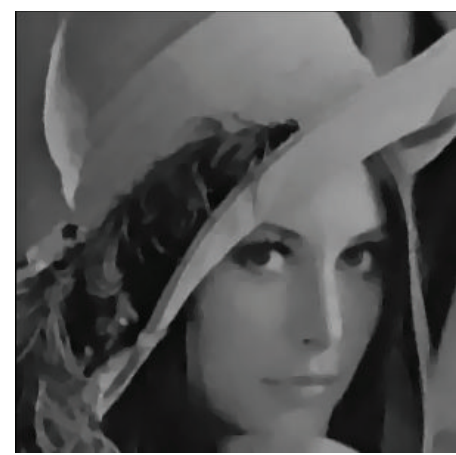

(e) The proposed method in [4]

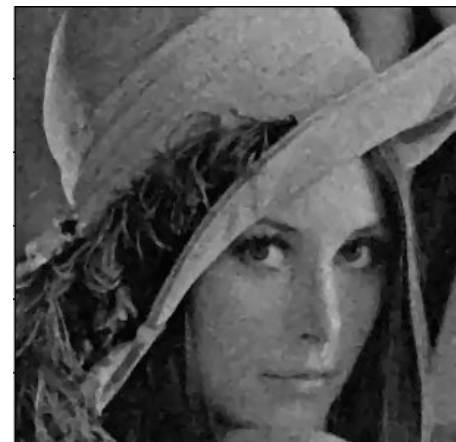

(c) Classical TV denoising

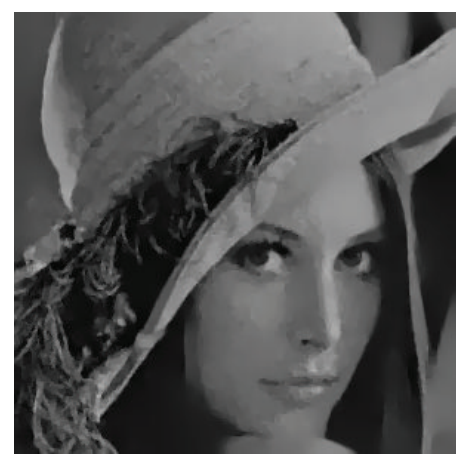

(f) The proposed method in this paper

FIGURE 2: Comparison of Gaussian noisy image and denoising results by different methods. (The SNR value of this comparison is as follows: noisy image SNR $=7.5325$, classical TV denoising SNR $=13.5687$, bilateral filter denoising SNR $=13.3146$, the proposed method in [4] SNR $=$ 14.1413, and the proposed method in this paper $\mathrm{SNR}=14.2835$.)

TABLE 1: Comparison of denoising results by different methods in terms of SNR value.

\begin{tabular}{lccccc}
\hline & Salt and pepper noise & Gaussian noise & Random noise & Real noisy image & Average SNR \\
\hline Noisy image & 8.1867 & 7.5325 & 7.1122 & 6.3736 & 7.3013 \\
Classical TV & 15.2426 & 13.5687 & 14.3264 & 11.4326 & 13.6425 \\
Bilateral filter & 15.0410 & 13.3146 & 14.2720 & 11.2724 & 13.4750 \\
Method in [4] & 15.7141 & 14.1413 & 14.6837 & 11.7538 & 14.0732 \\
Method proposed in this paper & 15.9832 & 14.2835 & 14.8013 & 12.1015 \\
\hline
\end{tabular}

4.2. Real Noisy Image. The comparable experiments provided in Section 4.1 reflect favorite denoising result of the proposed method in this paper. In the following experiment, a real noisy image experiment would be provided in this section. From this way, the denoising method proposed in this paper can be considered more effective and available in common applications. In order to better reflect the impaction of noise, we use camera directly for the display of computer. The main content of shoot image is text, including a large number of edges and detailed information. These experimental results are shown in Figure 4. The comparable denoising methods are the same as those in Section 4.1.

In Figure 4(a) is original noisy image, Figure 4(b) is denoising result by classical TV method, its visual effect is bad, Figure 4(c) is the denoising result by bilateral filter, it obviously contained much noise, and the denoising result of Figure $4(\mathrm{~d})$ is a little smoothing. Figure $4(\mathrm{e})$ is local part of denoising result by the proposed method in this paper, here noise is well removed, and the edge of text is well preserved, so that the visual effect is better than other methods.

In above experiments including three different noises, pepper and salt noise is a pulse noise which approximately is equal to amplitude but is randomly distributed in image; there are some clean points and contaminated points. Gaussian noise is almost equally distributed in each part of an image. The random noise is also known as background noise, which is the accumulation of a lot of random fluctuations caused by time; the value is unpredictable. The different noises have various characteristics, so many denoising methods can effectively reduce one kind but cannot deal with the others. Besides subjective visual effect, with the aim of deeply discussing the experiments and further show the effectiveness of the proposed methods, the objective SNR values of different denoising methods are also given in Table 1. From the average of SNR value provided in Table 1, we can see the average SNR value of proposed method is the highest; it rises about 


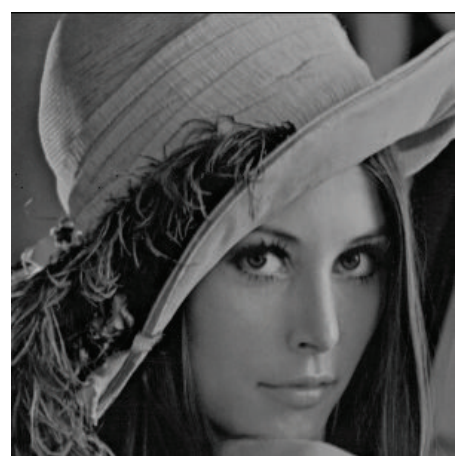

(a) Original Lena image

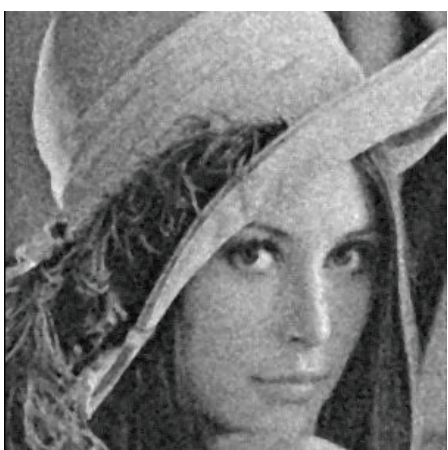

(d) Bilateral filter denoising

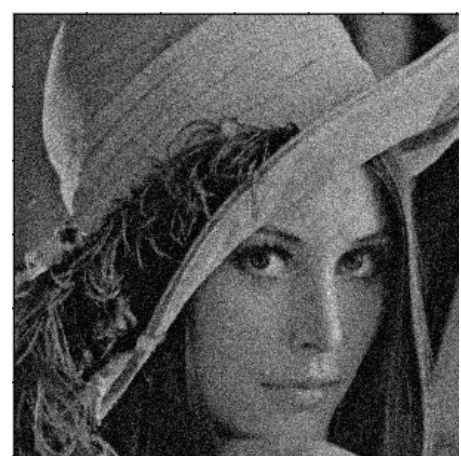

(b) Noisy image

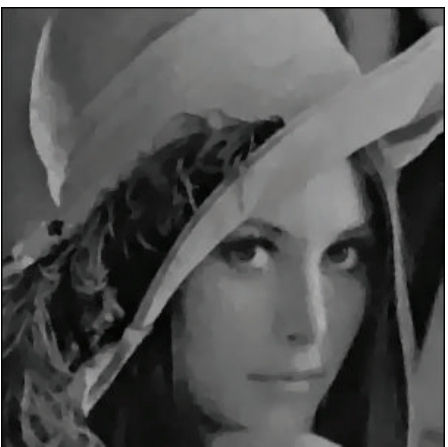

(e) The proposed method in [4]

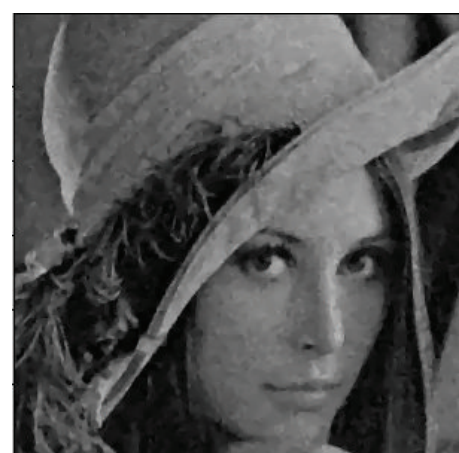

(c) Classical TV denoising

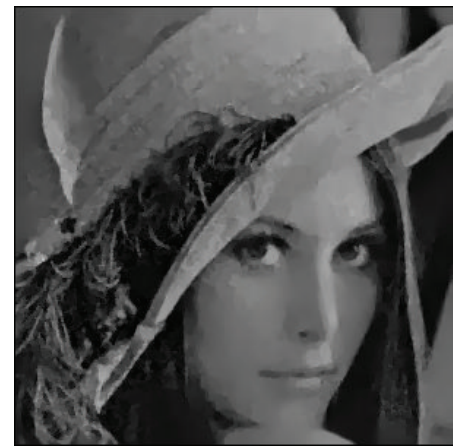

(f) The proposed method in this paper

FIGURE 3: Comparison of denoising results by different methods. (The SNR value of this comparison is as follows: noisy image SNR $=7.1122$, classical TV denoising SNR $=14.3264$, bilateral filter denoising SNR $=14.2720$, the proposed method in [4] SNR $=14.6837$, and the proposed method in this paper SNR = 14.8013.)

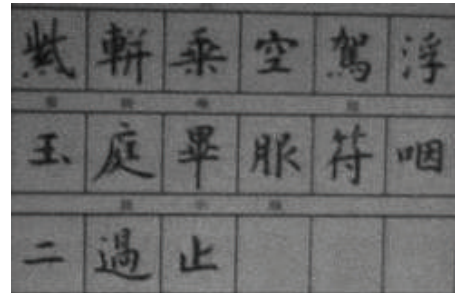

(a) Original Lena image

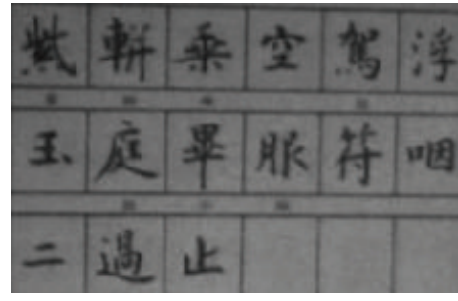

(b) Classical TV denoising

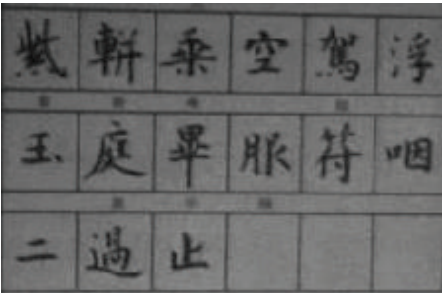

(c) Bilateral filter denoising

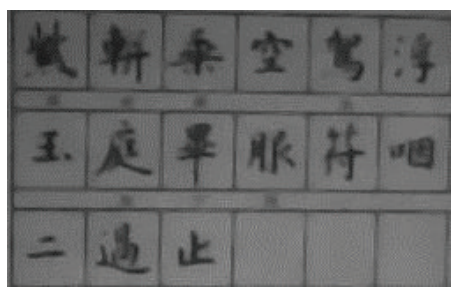

(d) The proposed method in [4]

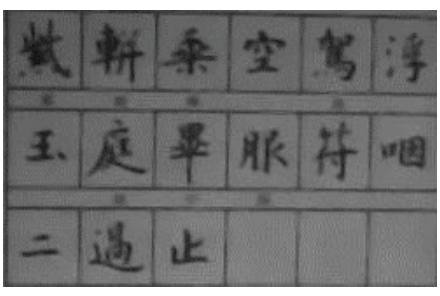

(e) The proposed method in this paper

FIGURE 4: Comparison of different denoising methods of real noisy image. (The SNR value of this comparison is as follows: noisy image SNR $=6.3736$, classical TV denoising SNR $=11.4326$, bilateral filter denoising SNR $=11.2724$, the proposed method in [4] SNR $=11.7538$, and the proposed method in this paper SNR $=12.1015$.)

0.3-0.6 dB compared to other referred methods. From the comparable results in Table 1 , it also can be seen that the objective result is consistent with the subjective value. The result of best visual effect also has the highest SNR value.
Based on the subjective visual effect and objective SNR value in simulated noisy and real noisy experiments, it can be seen that the denoising result by bilateral filter is worse than other methods. Above several comparable methods use 
global and fixed denoising pattern, which results in some details being missing and oversmoothing. The denoising method proposed in this paper is more effective and robust than other several methods. According to characteristic of different noisy image, the proposed method can adaptively adjust the regularization factor $\lambda$, the solution of $\lambda$ through iteration process, and each iteration updates the value of $\lambda$ by input image and current denoising image, so as to achieve the optimal regularization factor $\lambda$. Furthermore, the appropriate $\lambda$ can better balance the fidelity term and regularization term and then ensure reliable reduction of noise as well as protecting more fine details in image.

\section{Conclusion}

The widely used denoising algorithm based on global energy constraint can obtain good denoising effect on the simple structural image. But these methods can easily lose key detailed information when removing noise. For these reasons, an image denoising method based on local adaptive regularization is proposed in this paper, it can effectively control denoising degree in different area of noisy image by constraining residual local energy of energy function, and state-of-the-art denoising result can be obtained by this way. In this method, detailed information of image can also be well preserved at the process of denoising. From experiments of simulated and real noisy image, it is proved that the proposed denoising method is effective and robust. However, there are still some drawbacks in this method, such as time complexity. All of these experiments are operated on $4 \mathrm{M}$ computer and platform is Matlab. For an $800 * 600$ image, the consuming time is $25 \mathrm{~S}$. It means that the time complexity of the proposed method is high, although the visual effect outweighs other methods. Therefore, we would focus on the improvement of time complexity of the proposed method and then inspire new insights for the further research in this aspect.

\section{Conflict of Interests}

The authors declare that there is no conflict of interests regarding the publication of this paper.

\section{Acknowledgments}

The work is supported by Science Research Program of Hubei Provincial Science \& Technology Department of China (no. 2015CFC781, no. 2014CFB612, and no. 2011cdb088), Culture \& Technology Promotion Program of China (no. 201307), Program of National Natural Science Foundation of China (nos. 61263030, 61463014), Innovation Projection of Culture Ministry (no. 201217), Ph.D. Technology Program (no. MY2014B018), and Program of Sichuan Provincial Department of Education (no. 15ZB0039).

\section{References}

[1] J. Weickert, "A review of nonlinear diffusion filtering," in ScaleSpace Theory in Computer Vision, vol. 1252, pp. 1-28, Springer, 1997.
[2] T. F. Chan and S. Esedoglu, "Aspects of total variation regularized $L^{1}$ function approximation,” Tech. Rep. 04-07, UCLA Mathematics Department, 2004.

[3] C. Bo and Z. Li, "Symmetric four order PDE denoising algorithm," Computer Engineering, vol. 34, no. 13, pp. 188-189, 2008.

[4] D. H. Xu and R. S. Wang, "Imaging denoising based on nonlocal regularization," The Research and Application of Computer, vol. 26, no. 12, pp. 4830-4832, 2009.

[5] M. D. Gupta and S. Kumar, "Non-convex P-norm projection for robust sparsity," in Proceedings of the 14th IEEE International Conference on Computer Vision (ICCV '13), pp. 1593-1600, December 2013.

[6] G. J. Liu and X. P. Zeng, "Map image adaptive regularization denoising," Journal of Chongqing University, vol. 35, no. 10, pp. 63-67, 2012.

[7] X. W. Liu and L. H. Huang, "A new nonlocal total variation regularization algorithm for image denoising," Mathematics and Computers in Simulation, vol. 97, no. 3, pp. 224-233, 2014.

[8] M. J. Chen, P. X. Yang, and J. Wang, "Adaptive image denoising model based regularization and TV fidelity," Journal of Chongqing Post and Telecommunication, vol. 23, no. 5, pp. 621625,2011

[9] S. Suman, "Image denoising using new adaptive based median filter," Signal \& Image Processing, vol. 5, no. 4, pp. 1-13, 2014.

[10] J. Yan and W. S. Lu, "Imaging denoising by generalized total variation regularization and least squares fidelity," Multidimensional Systems and Signal Processing, vol. 20, no. 1, pp. 89-97, 2015.

[11] A. Anilet and C. Hati, "Image denoising method using curvelet transform and wiener filter," International Journal of Advanced Research in Electrical, Electronics and Instrumentation Engineering, vol. 3, no. 1, pp. 6943-6950, 2014.

[12] Z. Liu, E. Blasch, Z. Xue, J. Zhao, R. Laganiere, and W. Wu, "Objective assessment of multiresolution fusion algorithms for context enhancement in night vision: a comparative study," IEEE Transactions on Pattern Analysis and Machine Intelligence, vol. 34, no. 1, pp. 94-109, 2011.

[13] L. I. Rudin, S. Osher, and E. Fatemi, "Nonlinear total variation based noise removal algorithms," Physica D, vol. 60, no. 1-4, pp. 259-268, 1992. 


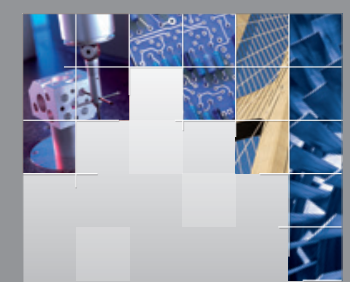

\section{Enfincering}
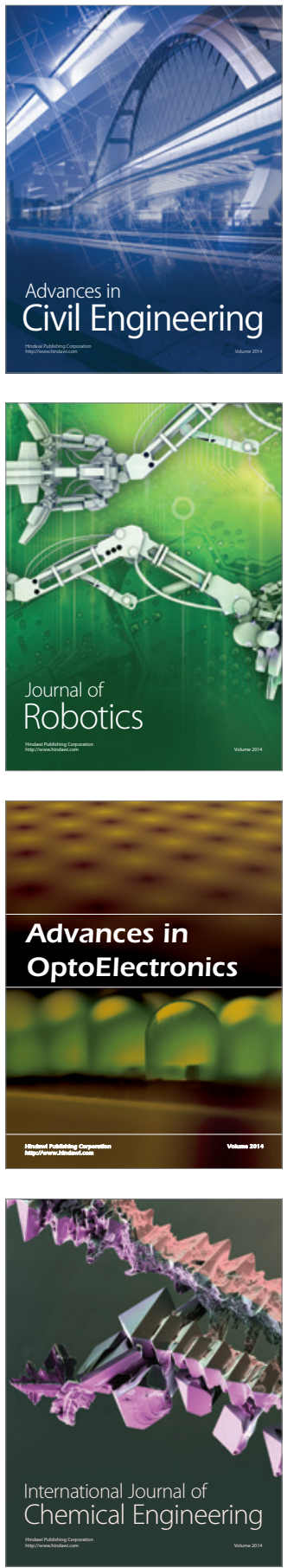

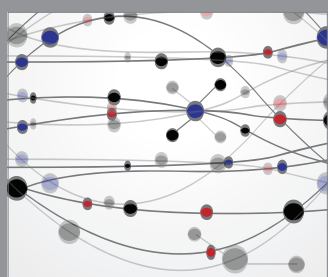

The Scientific World Journal

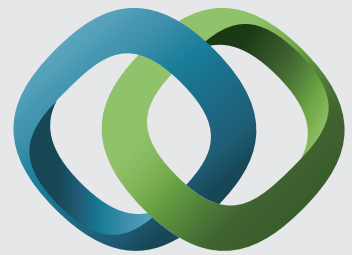

\section{Hindawi}

Submit your manuscripts at

http://www.hindawi.com
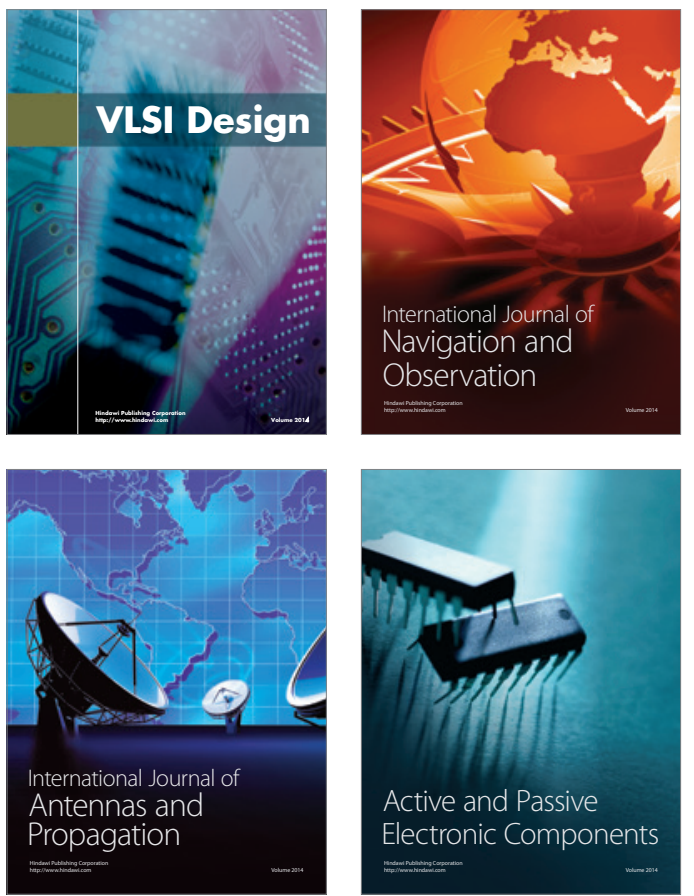
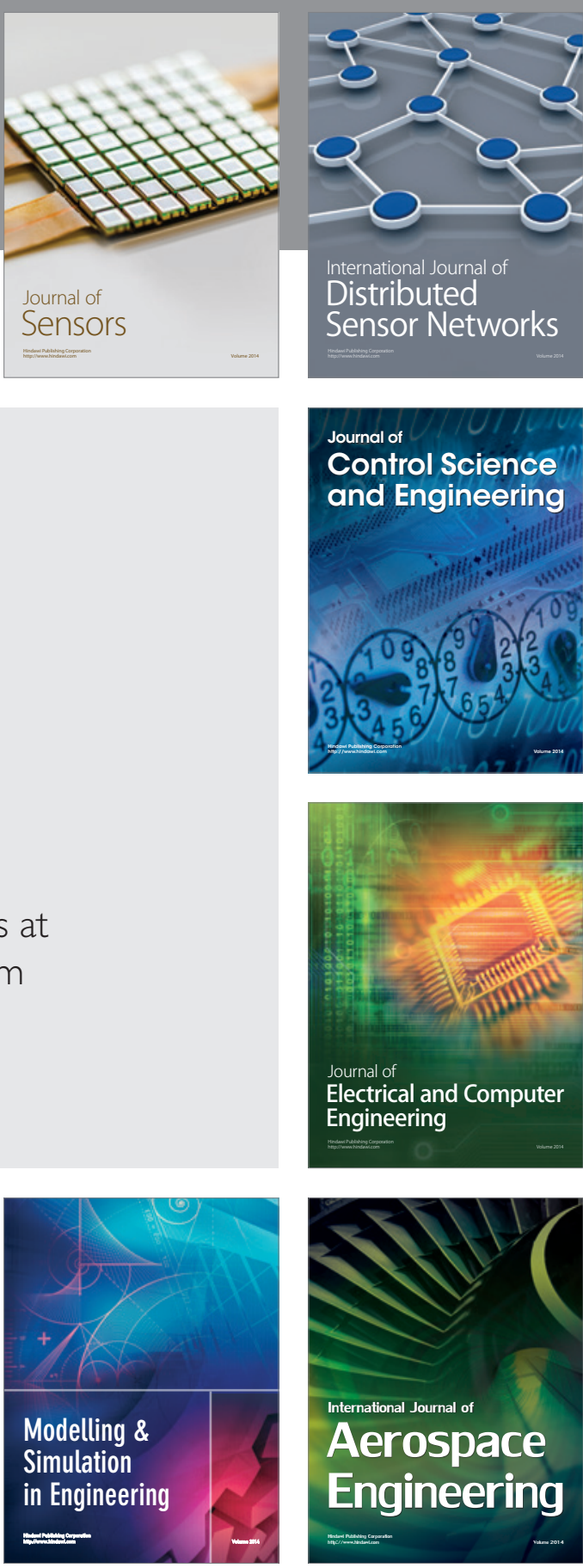

International Journal of

Distributed

Sensor Networks

Journal of

Control Science

and Engineering
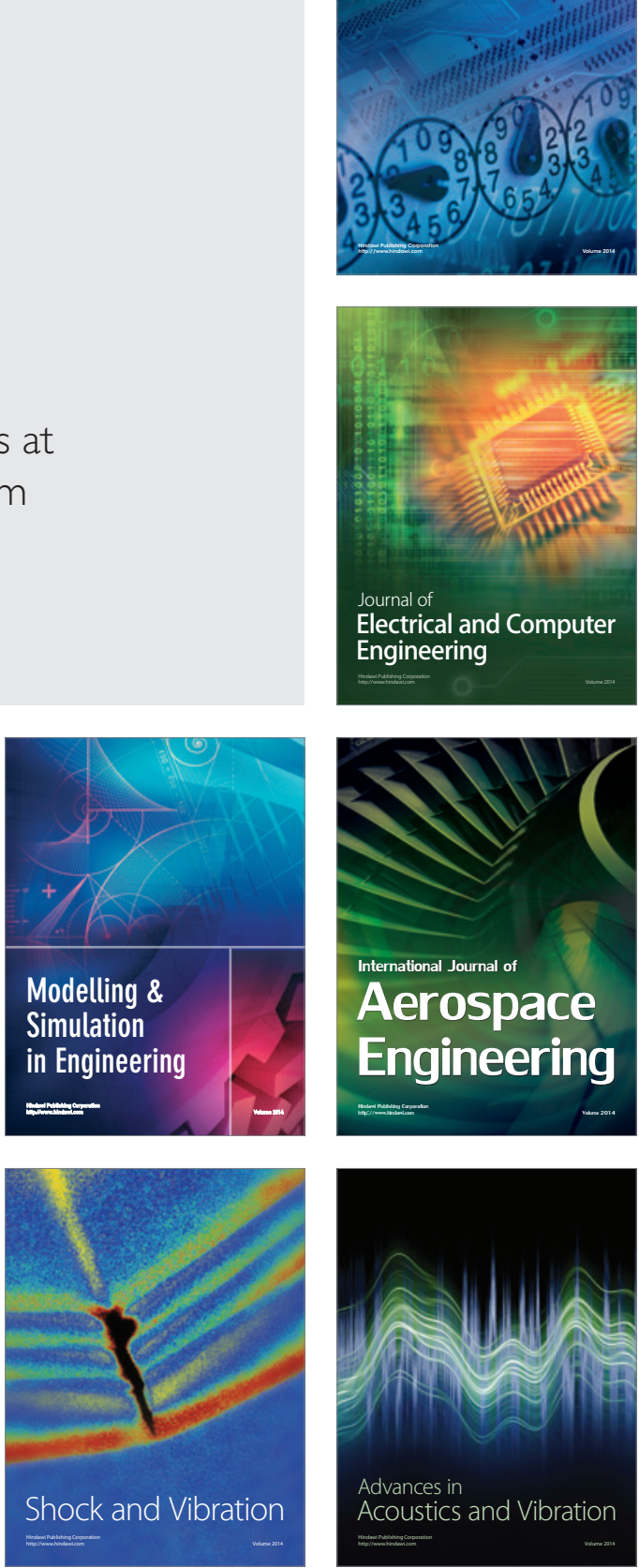